PROCEEDINGS OF THE

AMERICAN MATHEMATICAL SOCIETY

Volume 136, Number 12, December 2008, Pages 4175-4184

S 0002-9939(08)09639-1

Article electronically published on July 30, 2008

\title{
A NOTE ON THE $2 k$-TH POWER MEAN OF CHARACTER SUMS OVER THE QUARTER INTERVAL
}

\author{
ZHEFENG XU
}

(Communicated by Wen-Ching Winnie Li)

\begin{abstract}
We obtain an asymptotic formula for the $2 k$-th power mean of odd primitive character sums over the interval $\left[1, \frac{q}{4}\right)$.
\end{abstract}

\section{INTRODUCTION}

Let $q \geq 3$ be an integer, $\chi$ be a Dirichlet character modulo $q$. The various arithmetical properties of the character sums

$$
\sum_{a=N+1}^{N+H} \chi(a)
$$

were investigated by many authors; see [1], 2], 3], 4]. D.A. Burgess [5] obtained the mean value estimate

$$
\sum_{\chi \bmod }^{*} \sum_{q=1}^{p}\left|\sum_{m=1}^{h} \chi(n+m)\right|^{4} \leq 8 \tau^{7}(q) q^{2} h^{2},
$$

where $\sum_{\chi \bmod q}^{*}$ denotes the sum over all primitive characters modulo $q$ and $\tau(n)$ is the divisor function.

The author and Zhang [6] studied the $2 k$-th power mean of the even primitive character sums over the quarter interval $\left[1, \frac{q}{4}\right)$ by using mean value theorems of Dirichlet $L$-functions, and obtained the asymptotic formula as follows:

$$
\begin{aligned}
& \sum_{\chi(-1)=1}^{*}\left|\sum_{a<\frac{q}{4}} \chi(a)\right|^{2 k} \\
& =\frac{J(q) q^{k}}{16}\left(\frac{\pi}{8}\right)^{2 k-2} \prod_{p \mid q}\left(1-\frac{1}{p^{2}}\right)^{2 k-1} \prod_{p \nmid 2 q}\left(1-\frac{1-C_{2 k-2}^{k-1}}{p^{2}}\right)+O\left(q^{k+\epsilon}\right),
\end{aligned}
$$

Received by the editors November 8, 2007.

2000 Mathematics Subject Classification. Primary 11L40, 11L10; Secondary 11M38.

Key words and phrases. Odd primitive character sums, quarter interval, mean value, asymptotic formula.

The author was supported by NSF(10671155) of the People's Republic of China.

(C)2008 American Mathematical Society Reverts to public domain 28 years from publication 4175 
where $\sum_{\chi(-1)=1}^{*}$ denotes the sum over all primitive characters modulo $q$ such that $\chi(-1)=1, \epsilon$ is any fixed positive number, $J(q)$ denotes the number of all primitive characters modulo $q, \prod_{p \mid q}$ denotes the product over all prime divisors $p$ of $q$, and $C_{m}^{n}=\frac{m !}{n !(m-n) !}$. Because Lemma 3 of reference [8] is not correct, which will be explained in our Lemma 2.6, this result is also incorrect. The last factor in the main term $\left(1-\frac{1-C_{2 k-2}^{k-1}}{p^{2}}\right)$ should be $A(0, k, p, 2)$, as defined in our theorem below. However, this result is correct in the case $k=2$.

In this paper we study odd primitive character sums over the interval $\left[1, \frac{q}{4}\right)$ by transforming them to $L$-functions, and we obtain an asymptotic formula for the $2 k$-th power mean using the same method as in 6 .

Theorem 1.1. Let $q \geq 5$ be an odd integer. Then we have the asymptotic formula

$$
\begin{aligned}
& \sum_{\chi(-1)=-1}^{*}\left|\sum_{a<\frac{q}{4}} \chi(a)\right|^{2 k} \\
& =C(k) q^{k} J(q) \zeta^{2 k-1}(2) \prod_{p \mid q}\left(1-\frac{1}{p^{2}}\right)^{2 k-1} \prod_{p \nmid 2 q} A(0, k, p, 2)+O_{k}\left(q^{k+\epsilon}\right),
\end{aligned}
$$

where

$$
\begin{aligned}
C(k) & =\frac{1}{\pi^{2 k}} \sum_{i=0}^{k} C_{k}^{i}(-2)^{k-i} \sum_{j=0}^{i} 6^{j} \sum_{s=0}^{k-i} \sum_{t=0}^{i-j} \frac{A(|3 i+4 s-j-2 t-2 k|, k, 2,2)}{2^{|3 i+4 s-j-2 t-2 k|+2 k+1},} \\
A(m, k, p, s) & =\sum_{i=0}^{2 k-2} \frac{1}{p^{i s}} \sum_{j=0}^{i}(-1)^{j} C_{2 k-1}^{j} C_{k+m+i-j-1}^{m+i-j} C_{k+i-j-1}^{i-j}
\end{aligned}
$$

and $\zeta(s)$ denotes the Riemann zeta function.

Taking $k=2$ in our theorem and noting that

$$
\begin{aligned}
& \prod_{p \mid q}\left(1-\frac{1}{p^{2}}\right)^{3} \prod_{p \nmid 2 q}\left(1+\frac{1}{p^{2}}\right) \\
& =\prod_{p}\left(1+\frac{1}{p^{2}}\right) \prod_{p \mid q}\left(1-\frac{1}{p^{2}}\right)^{3} \prod_{p \mid 2 q} \frac{1}{1+\frac{1}{p^{2}}} \\
& =\frac{4}{5} \frac{\zeta(2)}{\zeta(4)} \prod_{p \mid q} \frac{\left(p^{2}-1\right)^{3}}{p^{4}\left(p^{2}+1\right)}
\end{aligned}
$$

$\zeta(2)=\frac{\pi^{2}}{6}$ and $\zeta(4)=\frac{\pi^{4}}{90}$, we get

Corollary 1.2. Let $q \geq 5$ be an odd integer. Then

$$
\sum_{\chi(-1)=-1}^{*}\left|\sum_{a<\frac{q}{4}} \chi(a)\right|^{4}=\frac{9}{128} q^{2} J(q) \prod_{p \mid q} \frac{\left(p^{2}-1\right)^{3}}{p^{4}\left(p^{2}+1\right)}+O\left(q^{2+\epsilon}\right) .
$$

Combining this with Corollary 2 of [6], we have the following 
Corollary 1.3. Let $q \geq 5$ be an odd integer. Then we have the asymptotic formula

$$
\sum_{\chi \bmod q}^{*}\left|\sum_{a<\frac{q}{4}} \chi(a)\right|^{4}=\frac{21}{256} q^{2} J(q) \prod_{p \mid q} \frac{\left(p^{2}-1\right)^{3}}{p^{4}\left(p^{2}+1\right)}+O\left(q^{2+\epsilon}\right) .
$$

The number $n>1$ is called square-full if in the prime factorization $n=p_{1}^{\alpha_{1}} p_{2}^{\alpha_{2}} \ldots$ $p_{r}^{\alpha_{r}}$ we have $\alpha_{i} \geq 2$ for all $i=1,2, \cdots, r$. Noting that $J(q)=\frac{\phi^{2}(q)}{q}$ if $q$ is a squarefull number, we have

Corollary 1.4. Let $q \geq 5$ be a square-full number with $2 \nmid q$. Then we have the asymptotic formula

$$
\sum_{\chi \bmod q}^{*}\left|\sum_{a<\frac{q}{4}} \chi(a)\right|^{4}=\frac{21}{256} q \phi^{2}(q) \prod_{p \mid q} \frac{\left(p^{2}-1\right)^{3}}{p^{4}\left(p^{2}+1\right)}+O\left(q^{2+\epsilon}\right) .
$$

Corollary 1.5. Let $p \geq 5$ be a prime. Then we have the asymptotic formula

$$
\sum_{\substack{\chi \bmod p \\ \chi \neq \chi_{0}}}\left|\sum_{a<\frac{p}{4}} \chi(a)\right|^{4}=\frac{21}{256} p^{3}+O\left(p^{2+\epsilon}\right) .
$$

Remark 1.6. From our theorem and the theorem in [6], we find that odd and even primitive characters have a very different contribution to the higher moment of character sums over the quarter interval. For the case $k=2$, from Corollaries 1.2 and 1.3, we see that the contribution of odd primitive characters is six times that of the even ones, although (see Remark 2.4 in the next section)

$$
\sum_{a=1}^{\left[\frac{q}{4}\right]} \chi(a)=0
$$

for some odd primitive characters, while

$$
\sum_{a=1}^{\left[\frac{q}{4}\right]} \chi(a) \neq 0
$$

for all even primitive characters.

\section{Some Lemmas}

To prove the theorem, we need the following lemmas.

Lemma 2.1. Let $\chi$ be a primitive character modulo $m$ with $\chi(-1)=-1$. Then we have

$$
\frac{1}{m} \sum_{b=1}^{m} b \chi(b)=\frac{i}{\pi} \tau(\chi) L(1, \bar{\chi}),
$$

where $\tau(\chi)=\sum_{a=1}^{m} \chi(a) e\left(\frac{a}{q}\right)$ is the Gauss sum, $e(y)=e^{2 \pi i y}$, and $L(s, \chi)$ denotes the Dirichlet L-function corresponding to $\chi$.

Proof. This can be easily deduced from Theorem 12.11 and Theorem 12.20 of [7]. 
Lemma 2.2. Let $q \geq 3$ be an odd number. For any nonprincipal character $\chi \bmod q$, we have

$$
\sum_{a=1}^{q} a \chi(a)=\frac{\chi(2) q}{1-2 \chi(2)} \sum_{a=1}^{\frac{q-1}{2}} \chi(a) .
$$

Proof. From the properties of Dirichlet characters, we have

$$
\begin{aligned}
\sum_{a=1}^{q} 2 a \chi(2 a) & =\sum_{a=1}^{\frac{q-1}{2}} 2 a \chi(2 a)+\sum_{a=\frac{q+1}{2}}^{q} 2 a \chi(2 a) \\
& =\sum_{a=1}^{\frac{q-1}{2}} 2 a \chi(2 a)+\sum_{a=1}^{\frac{q+1}{2}}(2 a-1) \chi(q+2 a-1)+q \sum_{a=1}^{\frac{q+1}{2}} \chi(2 a-1) \\
& =\sum_{a=1}^{q} a \chi(a)+q \sum_{a=1}^{\frac{q+1}{2}} \chi(2 a-1) .
\end{aligned}
$$

Noting that

$$
\sum_{a=1}^{\frac{q+1}{2}} \chi(2 a-1)+\sum_{a=1}^{\frac{q-1}{2}} \chi(2 a)=\sum_{a=1}^{q} \chi(a)=0
$$

we can write

$$
(1-2 \chi(2)) \sum_{a=1}^{q} a \chi(a)=\sum_{a=1}^{q} a \chi(a)-\sum_{a=1}^{q} 2 a \chi(2 a)=q \sum_{a=1}^{\frac{q-1}{2}} \chi(2 a)=\chi(2) q \sum_{a=1}^{\frac{q-1}{2}} \chi(a) .
$$

That is,

$$
\sum_{a=1}^{q} a \chi(a)=\frac{\chi(2) q}{1-2 \chi(2)} \sum_{a=1}^{\frac{q-1}{2}} \chi(a) .
$$

This proves Lemma 2.2.

Lemma 2.3. Let $q$ be an odd number and $\chi$ be a primitive Dirichlet character modulo $q$ such that $\chi(-1)=-1$. Then we have

$$
\sum_{a=1}^{\left[\frac{q}{4}\right]} \chi(a)=\frac{2+\bar{\chi}(2)-\bar{\chi}(4)}{2 i \pi} \tau(\chi) L(1, \bar{\chi})
$$

Remark 2.4. For even primitive characters $\chi$, by Lemma 2 of $[\underline{6}$,

$$
\sum_{a=1}^{\left[\frac{q}{4}\right]} \chi(a)=-\frac{i \bar{\chi}(4)}{2 \pi} \tau\left(\chi \chi_{4}\right) L\left(1, \bar{\chi} \chi_{4}\right)
$$

where $\chi_{4}$ is the primitive character modulo 4 . Since $\tau\left(\chi \chi_{4}\right) L\left(1, \bar{\chi} \chi_{4}\right) \neq 0$, we have

$$
\sum_{a=1}^{\left[\frac{q}{4}\right]} \chi(a) \neq 0
$$


for all even primitive characters. For odd primitive characters, from the identity in Lemma 2.3, we know that

$$
\sum_{a=1}^{\left[\frac{q}{4}\right]} \chi(a)=0
$$

if and only if $\chi(2)=-1$. For example, if $p$ is a prime, then the real character sum

$$
\sum_{a=1}^{\left[\frac{p-1}{4}\right]}\left(\frac{a}{p}\right)=0
$$

if and only if $p \equiv 3(\bmod 8)$. In this case, the number of quadratic residues and quadratic non-residues are equal in $\left[1, \frac{p-1}{4}\right]$.

Proof of Lemma 2.3. We separate $q$ into two cases: $q \equiv 1(\bmod 4)$ and $q \equiv 3$ $(\bmod 4)$. First, we suppose $q \equiv 1(\bmod 4)$. From the properties of the Dirichlet character modulo $q$, we can write

$$
\begin{aligned}
4 \chi(4) \sum_{a=1}^{q-1} a \chi(a)= & \sum_{a=1}^{\frac{q-1}{4}} 4 a \chi(4 a)+\sum_{a=\frac{q+3}{4}}^{\frac{2 q-2}{4}} 4 a \chi(4 a)+\sum_{a=\frac{2 q+2}{4}}^{\frac{3 q-3}{4}} 4 a \chi(4 a)+\sum_{a=\frac{3 q+1}{4}}^{q-1} 4 a \chi(4 a) \\
= & \sum_{a=1}^{\frac{q-1}{4}} 4 a \chi(4 a)+\sum_{a=1}^{\frac{q-1}{4}}(4 a+q-1) \chi(4 a-1) \\
& +\sum_{a=1}^{\frac{q-1}{4}}(4 a+2 q-2) \chi(4 a-2)+\sum_{a=1}^{\frac{q-1}{4}}(4 a+3 q-3) \chi(4 a-3) \\
= & \sum_{a=1}^{q-1} a \chi(a)+\chi(4) q \sum_{a=1}^{\frac{q-1}{4}} \chi(a-\overline{4}) \\
& +2 \chi(4) q \sum_{a=1}^{\frac{q-1}{4}} \chi(a-2 \cdot \overline{4})+3 \chi(4) q \sum_{a=1}^{\frac{q-1}{4}} \chi(a-3 \cdot \overline{4}) .
\end{aligned}
$$

Note that $\overline{4} \equiv \frac{3 q+1}{4}(\bmod q)$ if $q \equiv 1(\bmod 4)$. So from $(2.1)$, we have

$$
\begin{aligned}
4 \chi(4) \sum_{a=1}^{q-1} a \chi(a)= & \sum_{a=1}^{q-1} a \chi(a)-\chi(4) q \sum_{a=\frac{2 q+2}{4}}^{\frac{3 q-3}{4}} \chi(a) \\
& -2 \chi(4) q \sum_{a=\frac{q+3}{4}}^{\frac{2 q-2}{4}} \chi(a)-3 \chi(4) q \sum_{a=1}^{\frac{q-1}{4}} \chi(a) \\
= & \sum_{a=1}^{q-1} a \chi(a)-\chi(4) q \sum_{a=\frac{q+3}{4}}^{\frac{2 q-2}{4}} \chi(a)-3 \chi(4) q \sum_{a=1}^{\frac{q-1}{4}} \chi(a) \\
= & \sum_{a=1}^{q-1} a \chi(a)-\chi(4) q \sum_{a=1}^{\frac{q-1}{2}} \chi(a)-2 \chi(4) q \sum_{a=1}^{\frac{q-1}{4}} \chi(a),
\end{aligned}
$$


where we used the fact $\chi(-1)=-1$ and

$$
\sum_{a=\frac{q+3}{4}}^{\frac{2 q-2}{4}} \chi(a)=-\sum_{a=\frac{2 q+2}{4}}^{\frac{3 q-3}{4}} \chi(a)
$$

Now, from (2.2) and Lemma 2.2, we get

$$
4 \chi(4) \sum_{a=1}^{q-1} a \chi(a)=\sum_{a=1}^{q-1} a \chi(a)-(\chi(2)-2 \chi(4)) \sum_{a=1}^{q-1} a \chi(a)-2 \chi(4) q \sum_{a=1}^{\frac{q-1}{4}} \chi(a) .
$$

That is,

$$
\sum_{a=1}^{\frac{q-1}{4}} \chi(a)=\frac{\bar{\chi}(4)-\bar{\chi}(2)-2}{2 q} \sum_{a=1}^{q-1} a \chi(a)=\frac{\bar{\chi}(4)-\bar{\chi}(2)-2}{2 q} \sum_{a=1}^{q} a \chi(a) .
$$

Then from Lemma 2.1, we have

$$
\sum_{a=1}^{\frac{q-1}{4}} \chi(a)=\frac{2+\bar{\chi}(2)-\bar{\chi}(4)}{2 i \pi} \tau(\chi) L(1, \bar{\chi}) .
$$

This proves Lemma 2.3 in the case of $q \equiv 1(\bmod 4)$. By the same method, we can also prove

$$
\sum_{a=1}^{\frac{q-3}{4}} \chi(a)=\frac{2+\bar{\chi}(2)-\bar{\chi}(4)}{2 i \pi} \tau(\chi) L(1, \bar{\chi})
$$

if $q \equiv 3(\bmod 4)$. Combining $(2.3)$ and $(2.4)$, we can immediately get

$$
\sum_{a=1}^{\left[\frac{q}{4}\right]} \chi(a)=\frac{2+\bar{\chi}(2)-\bar{\chi}(4)}{2 i \pi} \tau(\chi) L(1, \bar{\chi})
$$

This completes the proof of Lemma 2.3.

Lemma 2.5. Let $f(x)$ be a polynomial of degree $k$ with leading coefficient $a_{0}$, and define the difference operator $\triangle$ by $(\triangle f)(x)=f(x)-f(x-1)$. Then we have

$$
\triangle^{k} f(x)=k ! a_{0}, \quad \triangle^{l} f(x)=0 \quad(l \geq k+1) .
$$

Proof. This can be easily deduced by the definition of the difference operator and mathematical induction.

Lemma 2.6. Let $q$ be an integer with $q>2$ and let $\tau_{k}(n)$ denote the $k$-th divisor function (i.e., the number of solutions of the equation $n_{1} n_{2} \cdots n_{k}=n$ in positive integers $\left.n_{1}, n_{2}, \cdots, n_{k}\right)$. Then for any complex variable $s$ with $\operatorname{Re}(s)>1$, we have the identity

$$
\sum_{\substack{n=1 \\(n, q)=1}}^{\infty} \frac{\tau_{k}^{2}(n)}{n^{s}}=\zeta^{2 k-1}(s) \prod_{p \mid q}\left(1-\frac{1}{p^{s}}\right)^{2 k-1} \prod_{p \nmid q} A(0, k, p, s) .
$$


Proof. This is Lemma 3 of 8 . But the result in 8 is not correct. From the Euler product formula and noting that (see formula 6.4.12 of [9])

$$
\tau_{k}(n)=\prod_{j=1}^{r} C_{k+\alpha_{j}-1}^{\alpha_{j}}
$$

if $n=p_{1}^{\alpha_{1}} p_{2}^{\alpha_{2}} \cdots p_{r}^{\alpha_{r}}$ is the factorization of $n$ into prime powers, we have

$$
\sum_{\substack{n=1 \\(n, q)=1}}^{\infty} \frac{\tau_{k}^{2}(n)}{n^{s}}=\prod_{p \nmid q} S(k, p, s),
$$

where

$$
S(k, p, s)=1+\frac{\left(C_{k}^{1}\right)^{2}}{p^{s}}+\frac{\left(C_{k+1}^{2}\right)^{2}}{p^{s}}+\cdots+\frac{\left(C_{k+i-1}^{i}\right)^{2}}{p^{s}}+\cdots .
$$

Now we calculate $S(k, p, s)$. For convenience, let $f(i)=\left(C_{k+i-1}^{i}\right)^{2}$. From the definition of the difference operator $\triangle$ in Lemma 2.5, we have

$$
\begin{aligned}
& S(k, p, s)\left(1-\frac{1}{p^{s}}\right)=1+\frac{\triangle f(1)}{p^{s}}+\frac{\triangle f(2)}{p^{2 s}}+\cdots+\frac{\triangle f(i)}{p^{i s}}+\cdots, \\
& S(k, p, s)\left(1-\frac{1}{p^{s}}\right)^{2}=1+\frac{\triangle f(1)-f(0)}{p^{s}}+\frac{\triangle^{2} f(2)}{p^{2 s}}+\cdots+\frac{\triangle^{2} f(i)}{p^{i s}}+\cdots .
\end{aligned}
$$

So, from the binomial expansion theorem and comparing the coefficients of $p^{i s}$ for $1 \leq i \leq 2 k-2$, we have

$$
S(k, p, s)\left(1-\frac{1}{p^{s}}\right)^{2 k-1}=S^{\prime}(k, p, s)+\frac{\triangle^{2 k-1} f(2 k-1)}{p^{(2 k-1) s}}+\frac{\triangle^{2 k} f(2 k)}{p^{2 k s}}+\cdots ;
$$

here

$$
S^{\prime}(k, p, s)=\sum_{i=0}^{2 k-2} \frac{1}{p^{i s}} \sum_{j=0}^{i}(-1)^{j} C_{2 k-1}^{j} f(i-j) .
$$

Noting that $f(i)$ is a polynomial in $i$ of degree $2 k-2$, so from Lemma 2.5 we know that $\triangle^{2 k-1} f(2 k-1)=\triangle^{2 k} f(2 k)=\cdots=0$. This yields

$$
\begin{aligned}
S(k, p, s) & =\left(1-\frac{1}{p^{s}}\right)^{1-2 k} \sum_{i=0}^{2 k-2} \frac{1}{p^{i s}} \sum_{j=0}^{i}(-1)^{j} C_{2 k-1}^{j}\left(C_{k+i-j-1}^{i-j}\right)^{2} \\
& =\left(1-\frac{1}{p^{s}}\right)^{1-2 k} A(0, k, p, s) .
\end{aligned}
$$

Hence, Lemma 2.6 follows immediately from the fact $\zeta(s)=\prod_{p}\left(1-\frac{1}{p^{s}}\right)^{-1}$.

Lemma 2.7. Let $q$ be any odd integer with $q>2$ and $m \geq 0$ a fixed integer. Then for any complex variable $s$ with $\operatorname{Re}(s)>1$, we have the identity

$$
\sum_{\substack{n=1 \\(n, q)=1}}^{\infty} \frac{\tau_{k}\left(2^{m} n\right) \tau_{k}(n)}{n^{s}}=A(m, k, 2, s) \zeta^{2 k-1}(s) \prod_{p \mid q}\left(1-\frac{1}{p^{s}}\right)^{2 k-1} \prod_{p \nmid 2 q} A(0, k, p, s) .
$$

This lemma is a generalization of Lemma 2.6, which is just the case $m=0$. 
Proof. Noting that $\tau_{k}(n)$ is a multiplicative function, we can write

$$
\begin{aligned}
\sum_{\substack{n=1 \\
(n, q)=1}}^{\infty} \frac{\tau_{k}\left(2^{m} n\right) \tau_{k}(n)}{n^{s}} & =\tau_{k}\left(2^{m}\right) \sum_{\substack{n=1 \\
(n, q)=1}}^{\infty \nmid n} \frac{\tau_{k}^{2}(n)}{n^{s}}+\sum_{\substack{n=1 \\
(n, q)=1}}^{\infty} \frac{\tau_{k}\left(2^{m} n\right) \tau_{k}(n)}{n^{s}} \\
& =\tau_{k}\left(2^{m}\right) \sum_{\substack{n=1 \\
(n, q)=1}}^{\infty} \frac{\tau_{k}^{2}(n)}{n^{s}}+\sum_{\substack{r=1 \\
(r, q)=1 \\
2 \nmid r}}^{\infty} \sum_{j=1}^{\infty} \frac{\tau_{k}\left(2^{m+j}\right) \tau_{k}\left(2^{j}\right) \tau_{k}^{2}(r)}{\left(r \cdot 2^{j}\right)^{s}} \\
& =\left(\sum_{j=0}^{\infty} \frac{\tau_{k}\left(2^{m+j}\right) \tau_{k}\left(2^{j}\right)}{2^{s j}}\right) \sum_{\substack{n=1 \\
(n, q)=1 \\
2 \nmid n}}^{\infty} \frac{\tau_{k}^{2}(n)}{n^{s}} .
\end{aligned}
$$

For $n>1$, let $n=p_{1}^{\alpha_{1}} p_{2}^{\alpha_{2}} \cdots p_{r}^{\alpha_{r}}$ be the factorization of $n$ into prime powers. Then we have

$$
\sum_{\substack{n=1 \\(n, q)=1}}^{\infty} \frac{\tau_{k}\left(2^{m} n\right) \tau_{k}(n)}{n^{s}}=\left(\sum_{j=0}^{\infty} \frac{C_{k+m+j-1}^{m+j} C_{k+j-1}^{j}}{2^{s j}}\right) \sum_{\substack{n=1 \\(n, q)=1 \\ 2 \nmid n}}^{\infty} \frac{\tau_{k}^{2}(n)}{n^{s}} .
$$

Let

$$
S=\sum_{j=0}^{\infty} \frac{C_{k+m+j-1}^{m+j} C_{k+j-1}^{j}}{2^{s j}} .
$$

Now using the same method as calculating $S(k, p, s)$ in Lemma 2.6, we can also get

$$
\begin{aligned}
S & =\left(1-\frac{1}{2^{s}}\right)^{1-2 k} \sum_{i=0}^{2 k-2} \frac{1}{2^{i s}} \sum_{j=0}^{i}(-1)^{j} C_{2 k-1}^{j} C_{k+m+i-j-1}^{m+i-j} C_{k+i-j-1}^{i-j} \\
& =\left(1-\frac{1}{2^{s}}\right)^{1-2 k} A(m, k, 2, s) .
\end{aligned}
$$

Noting that

$$
\sum_{\substack{n=1 \\(n, q)=1 \\ 2 \nmid n}}^{\infty} \frac{\tau_{k}^{2}(n)}{n^{s}}=\sum_{\substack{n=1 \\(n, 2 q)=1}}^{\infty} \frac{\tau_{k}^{2}(n)}{n^{s}}
$$

if $q$ is odd, we can easily get Lemma 2.7 from (2.5), (2.6) and Lemma 2.6.

Lemma 2.8. Let $q$ be any odd integer with $q>2, \chi$ be the Dirichlet character modulo $q$ and $m \geq 1$ be a fixed integer. Then we have the following asymptotic formulas:

$$
\sum_{\chi(-1)=-1}^{*} \chi\left(2^{m}\right)|L(1, \bar{\chi})|^{2 k}=\frac{J(q)}{2^{m+1}} \sum_{\substack{n=1 \\(n, q)=1}}^{\infty} \frac{\tau_{k}\left(2^{m} n\right) \tau_{k}(n)}{n^{2}}+O\left(q^{\epsilon}\right)
$$


and

$$
\sum_{\chi(-1)=-1}^{*} \bar{\chi}\left(2^{m}\right)|L(1, \bar{\chi})|^{2 k}=\frac{J(q)}{2^{m+1}} \sum_{\substack{n=1 \\(n, q)=1}}^{\infty} \frac{\tau_{k}\left(2^{m} n\right) \tau_{k}(n)}{n^{2}}+O\left(q^{\epsilon}\right) .
$$

Proof. By using the same method as in proving Lemma 5 of [6], we can get these formulas.

\section{Proof of the theorem}

In this section we will complete the proof of the theorem. It is well known that $|\tau(\chi)|=\sqrt{q}$ if $\chi$ is a primitive character. So from Lemma 2.3, we can write

$$
\begin{aligned}
& \sum_{\chi(-1)=-1}^{*}\left|\sum_{a<\frac{q}{4}} \chi(a)\right|^{2 k} \\
= & \frac{q^{k}}{(2 \pi)^{2 k}} \sum_{\chi(-1)=-1}^{*}|2+\bar{\chi}(2)-\bar{\chi}(4)|^{2 k}|L(1, \bar{\chi})|^{2 k} \\
= & \frac{q^{k}}{(2 \pi)^{2 k}} \sum_{\chi(-1)=-1}^{*}[6+\bar{\chi}(2)+\chi(2)-2(\bar{\chi}(4)+\chi(4))]^{k}|L(1, \bar{\chi})|^{2 k} \\
= & \frac{q^{k}}{(2 \pi)^{2 k}} \sum_{\chi(-1)=-1}^{*} \sum_{i=0}^{k} C_{k}^{i}(6+\bar{\chi}(2)+\chi(2))^{i}(-2)^{k-i}(\bar{\chi}(4)+\chi(4))^{k-i}|L(1, \bar{\chi})|^{2 k} \\
= & \frac{q^{k}}{(2 \pi)^{2 k}} \sum_{i=0}^{k} C_{k}^{i}(-2)^{k-i} \sum_{j=0}^{i} 6^{j} \sum_{s=0}^{k-i} \sum_{t=0}^{i-j} \sum_{\chi(-1)=-1}^{*} \chi^{3 i+4 s-j-2 t-2 k}(2)|L(1, \bar{\chi})|^{2 k}
\end{aligned}
$$

where $\chi^{-1}$ has the same meaning as $\bar{\chi}$, the conjugate of the character $\chi$. Then from Lemma 2.8 and Lemma 2.7, we easily get

$$
\begin{aligned}
& \sum_{\chi(-1)=-1}^{*}\left|\sum_{a<\frac{q}{4}} \chi(a)\right|^{2 k} \\
& =C(k) q^{k} J(q) \zeta^{2 k-1}(2) \prod_{p \mid q}\left(1-\frac{1}{p^{2}}\right)^{2 k-1} \prod_{p \nmid 2 q} A(0, k, p, 2)+O_{k}\left(q^{k+\epsilon}\right),
\end{aligned}
$$

where

$$
\begin{gathered}
C(k)=\frac{1}{\pi^{2 k}} \sum_{i=0}^{k} C_{k}^{i}(-2)^{k-i} \sum_{j=0}^{i} 6^{j} \sum_{s=0}^{k-i} \sum_{t=0}^{i-j} \frac{A(|3 i+4 s-j-2 t-2 k|, k, 2,2)}{2^{|3 i+4 s-j-2 t-2 k|+2 k+1}}, \\
A(m, k, p, s)=\sum_{i=0}^{2 k-2} \frac{1}{p^{i s}} \sum_{j=0}^{i}(-1)^{j} C_{2 k-1}^{j} C_{k+m+i-j-1}^{m+i-j} C_{k+i-j-1}^{i-j} .
\end{gathered}
$$

This completes the proof of the theorem.

\section{ACKNOWLEDGMENT}

The author expresses his gratitude to the referee for very helpful and detailed comments. 


\section{REFERENCES}

1. G. Pólya, Über die Verteilung der quadratische Reste und Nichtreste, Göttingen Nachrichten, 1918, pp. 21-29.

2. I. M. Vinogradov, On the distribution of residues and non-residues of powers, Journal of the Physico-Mathematical Society of Perm. 1 (1918), 94-96.

3. A. V. Sokolovskiř, On a theorem of Sárkozy, Acta Arithmetica 41 (1982), 27-31. MR667707 (84e:10046)

4. M. Z. Garaev and A. A. Karatsuba, On character sums and the exceptional set of a congruence problem, Journal of Number Theory 114 (2005), 182-192. MR2163912 (2006f:11095)

5. D. A. Burgess, Mean value of character sums. II, Mathematika 33 (1987), 1-7. MR908834 (89g:11072)

6. Zhefeng $\mathrm{Xu}$ and Wenpeng Zhang, On the $2 k$ th power mean of the character sums over short intervals, Acta Arithmetica 121 (2006), 149-160. MR2216139(2006m:11122)

7. Tom M. Apostol, Introduction to Analytic Number Theory, Springer-Verlag, New York, 1976. MR0434929 (55:7892)

8. Wenpeng Zhang, Yuan Yi and Xiali He, On the $2 k$-th power mean of Dirichlet L-functions with the weight of general Kloosterman sums, Journal of Number Theory 84 (2000), 199-213. MR.1795790 (2002e:11115)

9. Chengdong Pan and Chengbiao Pan, Element of Analytic Number Theory, Science Press, Beijing, 1999, pp. 95.

Department of Mathematics, Northwest University, Xi'an, Shaanxi, People's RepubLIC OF CHINA

E-mail address: zhefengxu@hotmail.com; zfxu@nwu.edu.cn 